\title{
Erector Spinae Plane Block for Perioperative Pain Control and Short-term Outcomes in Lumbar Laminoplasty: A Randomized Clinical Trial
}

\author{
Yanwu Jin' \\ Shanshan Zhao' \\ Jiahui $\mathrm{Cai}^{2}$ \\ Marcelle Blessing ${ }^{3}$ \\ Xin Zhao (D) \\ Haizhu $\operatorname{Tan}^{2}$ \\ Jinlei $\mathrm{Li} \mathbb{1}^{3}$
}

'Department of Anesthesiology, Second Hospital of Shandong University,

Shandong University, Jinan, Shandong,

People's Republic of China; ${ }^{2}$ Department of Preventive Medicine, Shantou University Medical College, Shantou, Guangdong, People's Republic of China; ${ }^{3}$ Department of Anesthesiology, Yale University School of Medicine, New Haven, CT, USA
Correspondence: Jinlei Li; Haizhu Tan Department of Anesthesiology, Yale University School of Medicine, 333 Cedar Street, TMP 3, New Haven, CT,

06520-805I, USA

Tel +l $203785-2802$

Fax + I 203 785-6664

Email jinlei.li@yale.edu; linnanqia@|26. com
Background: Spine surgery causes severe pain and can be associated with significant opioid utilization; however, the evidence for opioid-sparing analgesic techniques such as erector spinae plane (ESP) block from controlled studies is limited. We aimed to investigate the analgesic effects of ESP block in lumbar laminoplasty.

Methods: In this prospective, double-blind, controlled single-center trial, 62 consecutive elective lumbar laminoplasty patients were randomized into either a control group (Group G, $\mathrm{N}=32$ ) or a treatment group (Group E, N=30). Group $\mathrm{G}$ received general anesthesia and multimodal analgesia, similar to group E, while Group E received additional bilateral ESP block after induction of general anesthesia. The primary outcome was postoperative pain scores for the first $48 \mathrm{~h}$ after surgery, and the secondary outcomes analyzed included intraoperative anesthetic usage, perioperative analgesic consumption, return of bowel function and satisfaction for acute pain management indicated by overall benefit of analgesia score (OBAS).

Results: Significant differences in pain scores over time were found between the two groups $(P=0.010)$, with Group E patients having significantly lower pain scores than Group G during the first six hours $(P=0.000)$. The opioid consumption in Group $\mathrm{G}$ was significantly higher than in Group E both intraoperatively $(P=0.000)$ and postoperatively $(P=0.0005)$. Group E patients had lower intraoperative sevoflurane requirement, improved satisfaction with pain management, and earlier return of bowel function than Group G patients.

Conclusion: ESP block is effective in reducing postoperative pain scores and lowering opioid utilization (both intraoperatively and postoperatively), resulting in improved patient satisfaction for pain management in lumbar laminoplasty.

Keywords: erector spinae plane block, laminoplasty, spine, perioperative pain, opioid, shortterm outcome

\section{Introduction}

Spine surgery is frequently associated with patient dissatisfaction because of poorly controlled postoperative pain, chronic postoperative opioid dependence and longterm functional disability. ${ }^{1,2}$ Since its original description, erector spinae plane (ESP) block, an ultrasound-guided interfascial plane block with local anesthetics deposited between the transverse process and erector spinae muscle, ${ }^{3,4}$ is an emerging regional anesthesia technique that continues to gain popularity due its ease of performance and efficacy in pain control. ${ }^{5}$ In particular, ESP block, performed at the level of the lumbar spine, directly and consistently targets the dorsal rami of the spinal nerves ${ }^{1,2,4,6}$ and has been reported to provide 
postoperative analgesia in spine surgery in small case series, ${ }^{7}$ retrospective studies, ${ }^{8}$ and in small randomized controlled studies. ${ }^{1,2,9-11}$ Our primary hypothesis is that ESP block can improve perioperative analgesia with lower pain scores in lumbar laminoplasty patients. In secondary analysis, we also prospectively investigated the effects of the ESP block on intraoperative anesthetic requirements, perioperative opioid consumption, and patient satisfaction with pain management after lumbar spine surgery.

\section{Methods}

\section{Study Design}

This study is a single center, prospective, randomized, controlled clinical trial conducted with Institutional Review Board approval from the Second Hospital of Shandong University in China (number KYLL-2018(LW) 031), and the guidelines outlined in the Declaration of Helsinki were followed. Written informed consent was obtained from all study participants. The study was retrospectively registered at http://www.chictr.org.cn, identifier ChiCTR1900026706. No further data will be shared besides what is included in this manuscript.

\section{Patient Enrollment and Randomization}

Seventy-two consecutive patients for elective two- or three-level lumbar laminoplasty for lumbar spinal stenosis were identified from September 1, 2018 to May 31, 2019. Seventy partients were enrolled and randomly allocated into two groups using computer-generated randomization numbers contained in sealed opaque envelopes and two were excluded due to patient refusal. The follow-up for eight patients could not be included in the final statistical analysis: five patients experienced PCA device failure and three patients declined further follow-up postoperatively, resulting in 62 patients included in the final statistical analysis (Figure 1). Inclusion criteria: elective laminoplasty and American Society of Anesthesiologists (ASA) physical status I-III. Exclusion criteria: patient refusal, body mass index (BMI) less than 18 or higher than $35 \mathrm{~kg} / \mathrm{m}^{2}$, age less than 18 years old, pregnancy, history of relevant allergy to related perioperative medications, previous lumbar spine surgery, existing contraindications to nerve block such as coagulopathy, local and systemic infection, and inability to operate a patient-controlled analgesia (PCA) pump.

The same anesthesiologist administered the ultrasoundguided ESP block and general anesthetic for all patients.
The blocks were performed with the patient in the prone position under general anesthesia. All patients were blinded to their group assignments. The surgeons were not blinded to the group assignments; however, they were not involved in performing any of the postoperative assessments. Postoperative follow-up was performed by two certified nurses who were also blinded to the patient group assignments.

\section{Anesthesia, Analgesia and Ultrasound-guided ESP}

For all patients, ASA standard monitors were applied, and an arterial line was placed before induction of general anesthesia, following the standard protocol for patients undergoing lumbar spine surgery at the study institute. General anesthesia with endotracheal intubation was induced with propofol $(1.5 \sim 2.5 \mathrm{mg} / \mathrm{kg})$, sufentanil $(0.2 \sim 0.3 \mu \mathrm{g} / \mathrm{kg})$ and cisatracurium $(0.2 \mathrm{mg} / \mathrm{kg})$. Group $\mathrm{G}$ received general anesthesia and multimodal analgesia, while Group E received the same multimodal analgesia supplemented with pre-incision ultrasound-guided ESP blocks bilaterally. Block level was determined based on the vertebral levels of the surgery. For two-level surgery, the block was performed at the upper level and for threelevel surgery the block was performed at the middle level. The ESP block was performed after induction of general anesthesia and after patients were placed in the prone position. A 5-2 MHz low-frequency probe (C5-1B convex transducer, Wisonic Navi, Shenzhen, China) was first placed in a longitudinal orientation in the midline to identify the spinous process of the appropriate lumbar level, then moved 2-3 cm laterally until the paraspinal muscles and the transverse process at that level could be visualized (Figure 2). After standard sterilization, a 21-G×100 mm short bevel non-echogenic nerve block needle (PAJUNK Gmbh Medizintechnologie, Geisingen, Germany) was advanced in-plane under real-time ultrasound guidance, through skin, subcutaneous tissue and erector spinae muscle until reaching the transverse process. After negative aspiration for blood, a small volume of local anesthetic was injected to confirm the position of the needle tip between the erector spinae muscle and the transverse process. A total of $20 \mathrm{~mL}$ of $0.375 \%$ ropivacaine hydrochloride was incrementally injected with intermittent negative aspiration on each side. The duration from block completion to surgical incision was at least $30 \mathrm{~min}$ to allow sufficient time for ESP block to take effect. 


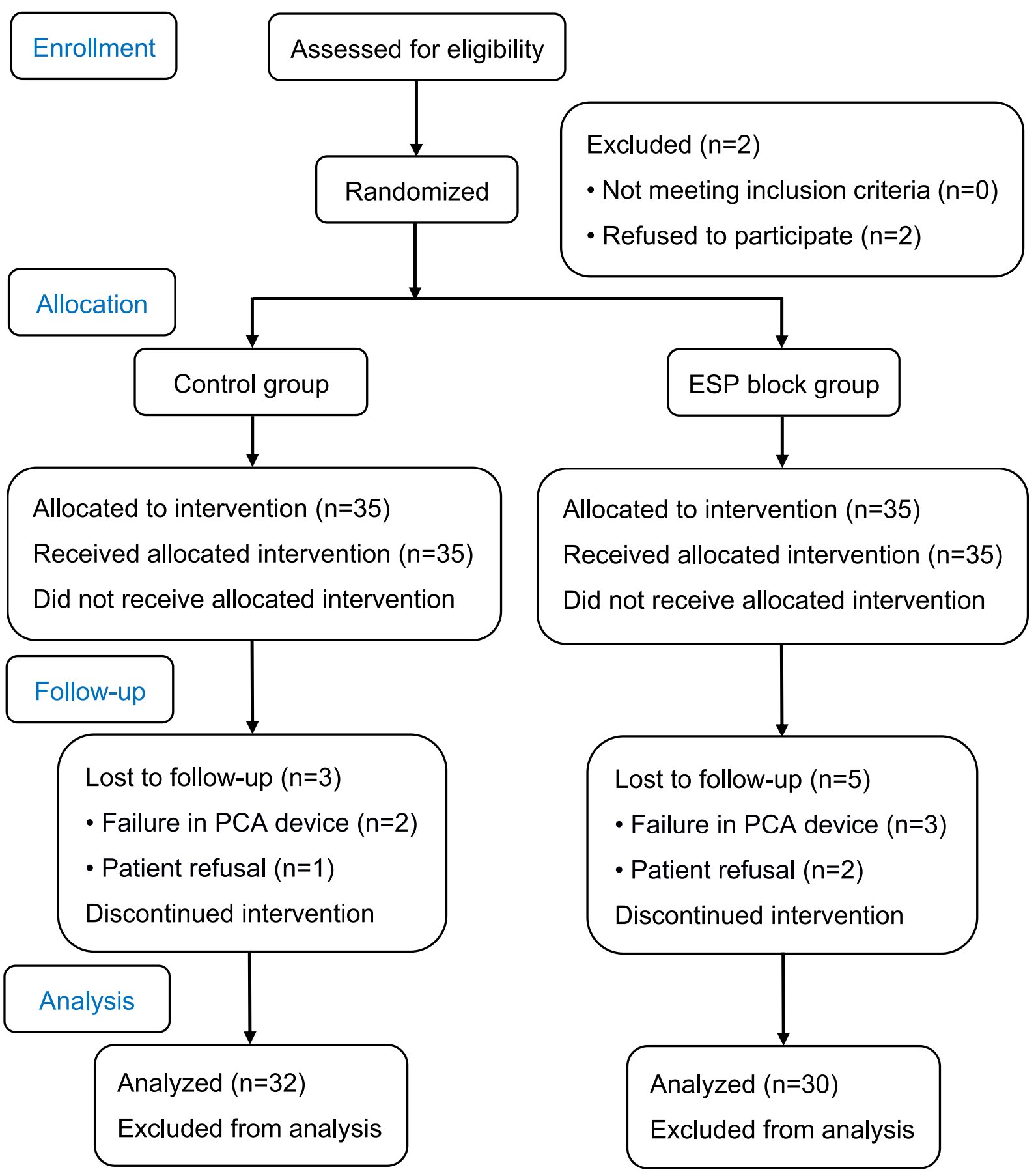

Figure I Flow diagram in CONSORT format.

Notes: Adapted from: Schulz KF, Altman DG, Moher D, CONSORT Group. CONSORT 2010 Statement: Updated Guidelines for Reporting Parallel Group Randomised Trials. PLoS Med. 2010;7(3):el00025I. Copyright: @ 2010 Schulz et al. Creative Commons Attribution License. https://journals.plos.org/plosmedicine/article?id=10.137I/ journal.pmed.100025I.

Abbreviations: ESP, Erector Spinae Block; PCA, patient-controlled analgesia.

Intraoperatively, maintenance of anesthesia was achieved with sevoflurane ( $1 \sim 4 \%$ in $60 \%$ oxygen); sevoflurane concentration was titrated to maintain the Bispectral Index (BIS) between 40 and 60. Sufentanil boluses $(5 \mu \mathrm{g})$ were administered every $10 \mathrm{~min}$ as needed to maintain blood pressure and heart rate within $20 \%$ of the patient's baseline upon arrival to the hospital. Cisatracurium $(0.1 \mathrm{mg} / \mathrm{kg} \bullet \mathrm{h})$ was administered 


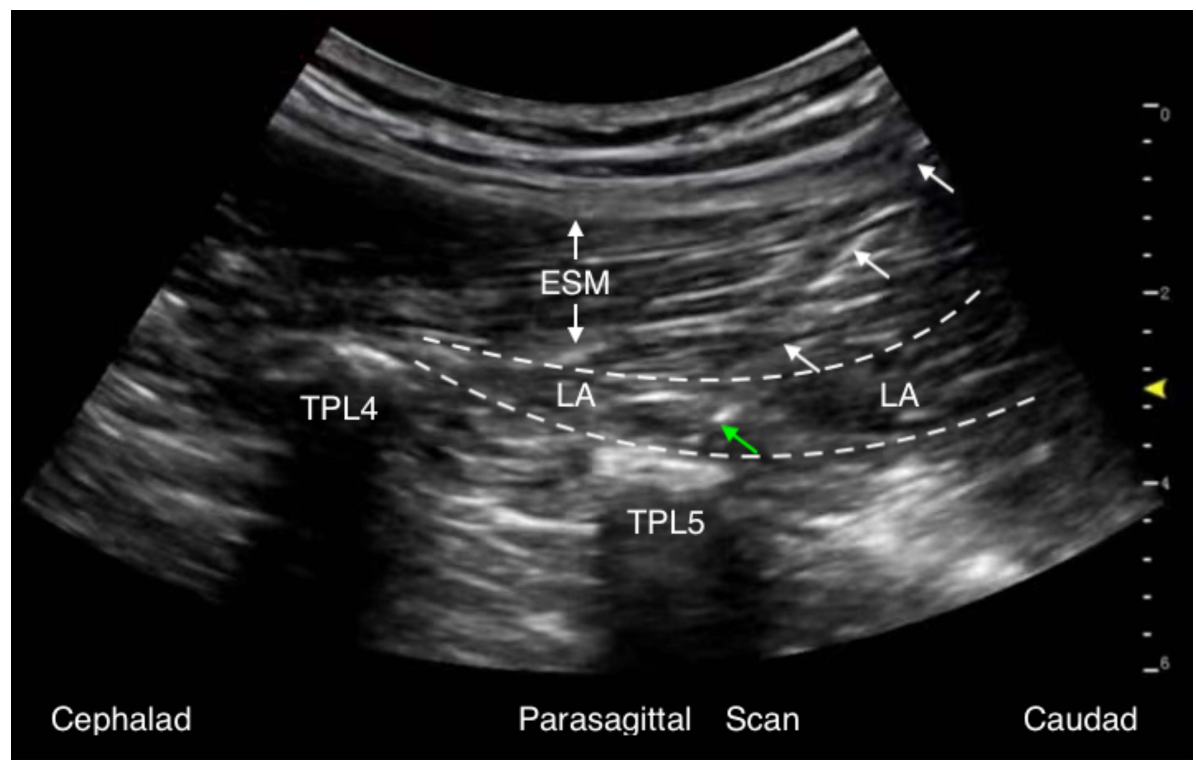

Figure 2 Sonographic illustration of erector spinae plane (ESP) block in parasagittal scan. White arrow, needle trajectory; green arrow, needle tip. Abbreviations: ESM, erector spinae muscle; LA, local anesthetic; TPL, lumbar transverse process.

during maintenance at the discretion of the anesthesiologist. Sufentanil dosing was switched to remifentanil infusion at the rate of $0.1-0.4 \mu \mathrm{g} / \mathrm{kg} / \mathrm{min}$ at the start of surgical closure to facilitate smooth extubation, titrated to maintain similar hemodynamics as sufentanil achieved. ${ }^{12,13}$

All patients were admitted to the Spine Surgery Postoperative Observational Unit (essentially a combination of postanesthesia care unit (PACU) and level two surgical step-down unit) for observation for the first $48 \mathrm{~h}$ after surgery before being transferred to a regular hospital floor. All patients were provided with a PCA pump that delivered a mixture of sufentanil $1 \mu \mathrm{g} / \mathrm{mL}$, dezocine $0.2 \mathrm{mg} / \mathrm{mL}$, dexmedetomidine $1 \mu \mathrm{g} / \mathrm{mL}$, and palonosetron $0.005 \mathrm{mg} / \mathrm{mL}$ at a basal rate of $2 \mathrm{~mL} / \mathrm{h}$, with a 2-mL demand dose at lock-out intervals of $15 \mathrm{~min}$. The PCA pump was started immediately after the operation upon arrival in the Spine Surgery Postoperative Observational Unit. Intravenous sodium parecoxib $40 \mathrm{mg}$ and intramuscular pethidine $50 \mathrm{mg}$ were the first- and second-line treatments (ordered to be administered $30 \mathrm{~min}$ after first-line treatment) for breakthrough pain respectively for visual analog scale (VAS) $\geq 4$. Breakthrough nausea and vomiting were managed with $0.25 \mathrm{mg}$ palonosetron intravenously when patients requested treatment, or patients were observed to have signs and/or symptoms of nausea and/or vomiting. Overall benefit of analgesia score (OBAS), a validated multidimensional instrument to assess pain intensity, opioid-related adverse effects such as distress from vomiting or itching, and patient satisfaction for pain control, ${ }^{14}$ was measured $24 \mathrm{~h}$ postoperatively in the Spine Surgery Postoperative Observational Unit.

The primary outcome was VAS pain score for the first $48 \mathrm{~h}$ after surgery. The secondary outcomes included intraoperative opioid and sevoflurane consumption, postoperative opioid consumption and number of postoperative PCA attempts at $0-12 \mathrm{~h}, 12-24 \mathrm{~h}$, 24-48 h, and 0-48 h, pain management satisfaction indicated by OBAS score at $24 \mathrm{~h}$ postoperatively, and postoperative rescue analgesic usage for the first 48 $\mathrm{h}$ after surgery. Frequency of adverse events such as nausea, vomiting, and dizziness were also measured as well as functional outcomes such as return of bowel function (indicated by the time to first postoperative flatus), length of hospital stay, and cost of hospitalization.

\section{Statistical Analysis}

The sample size calculation and all statistical data analysis was performed with $\mathrm{R}$ software. The significance level was set at a $P$-value $<0.05$.

To estimate the sample size and power, 40 patients were randomly assigned into two groups in the preliminary pilot study. For the primary outcome, VAS pain scores for the first $48 \mathrm{~h}$ after operation, we first applied the sample size formula for a two-sample longitudinal data to 
compute the predicted power using a two-sided test. The possible new enrolled sample size was obtained. We repeated this procedure until the predicted power achieved the preset power $\beta(\beta=0.8)$. For secondary outcomes (analgesic consumption), we again calculated the sample size and power under the hypothesis tested. Combining all predicted sample size together, the estimated sample size was about 28 for each group. Considering 10\% loss of follow-ups, the effective sample size was set at 36 for each group.

Data are presented as mean (SD), or median (interquartile range (IQR): 25 th percentile to 75 th percentile) for continuous variables, and frequency and percentage for categorical variables. The study for our primary outcome, VAS at $1,3,6,12,24$, and $48 \mathrm{~h}$ after operation, was a repeated measures design because multiple measurements were on the same unit and observed over time. ${ }^{15}$ However, VAS over times tended to correlate with each other. It was therefore important to account for the correlation between measurements. We applied Mauchly's Test of Sphericity to test the correlations between measurements over times. ${ }^{16}$

In equation (1),

$\mathrm{X}_{\mathrm{i}}$ where, $\mathrm{i}=1,2$

$Y_{j}$, where $\mathrm{j}=1,3,6,12,24,48$

$Z_{\mathrm{i}}$, Where,

$$
\begin{aligned}
& Z_{1}=\left(\begin{array}{llll}
1,2, & \ldots & 30
\end{array}\right)^{\mathrm{T}} \\
& Z_{2}=\left(\begin{array}{llll}
1,2, & \ldots & \ldots & 32
\end{array}\right)^{T}
\end{aligned}
$$

We let $Y_{j}$ be VAS over time, $X_{i}$ be types of anesthesia, $\mathrm{Z}$ be patients in each group. $\mathrm{i}$ be the number of group, and $\mathrm{j}$ be time interval.

From equation (1), we found that multivariate analysis of variance (MANOVA) could not be applied to analyze the difference of $Y_{j}$ between two groups. Ball Divergence Test $(\mathrm{BD})^{17}$ is a nonparametric two-sample test. It is a measure of the difference between two probability measures in separable Banach spaces. ${ }^{17}$ Hence, BD was adopted to identify the difference for VAS over time between the two groups. In this study, let $\mathrm{X}_{\mathrm{i}}$ and $\mathrm{Y}_{\mathrm{j}}$ to express two groups' patient's measurements of VAS overtimes.

$\mathrm{X}_{i}=\left(\mathrm{X}_{i, 1}, \mathrm{X}_{i, 3}, \mathrm{X}_{i, 6}, \mathrm{X}_{i, 12}, \mathrm{X}_{i, 24}, \mathrm{X}_{i, 48}\right), \quad$ where, $\quad \mathrm{i}=$ $1,2, \ldots \ldots, 30$

$Y_{j}=\left(\mathrm{Y}_{j, 1} \mathrm{Y}_{j, 3} \mathrm{Y}_{j, 6} \mathrm{Y}_{j, 12} \mathrm{Y}_{j, 24} \mathrm{Y}_{j, 48}\right)$, where, $\mathrm{j}=1.2, \ldots \ldots, 32$

Defined the distance between $\mathrm{X}_{i}$ 's and $\mathrm{Y}_{j}$ 's as the equation (2)

$$
d(A, B)=\sqrt{\begin{array}{l}
\left(A_{1}-B_{1}\right)^{2}+\left(A_{3}-B_{3}\right)^{2}+\left(A_{6}-B_{6}\right)^{2} \\
+\left(A_{12}-B_{12}\right)^{2}+\left(A_{24}-B_{24}\right)^{2} \\
+\left(A_{48}-B_{48}\right)^{2}
\end{array}}
$$

We used equation (2) to calculate the distance in our study as:

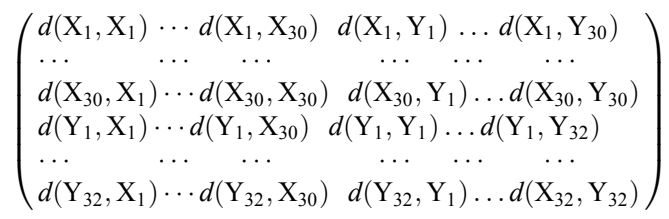

We subsequently compute the test statistic with techniques described by Pan et al. ${ }^{17}$

After comparing the VAS difference between the two groups using Ball Divergence Test, we subsequently studied the change pattern as well as the extreme points over time between the groups.

Two sample rank sum test was applied to analyze the cardinal variables with non-normal distribution (such as, intraoperative sevoflurane consumption, intraoperative inhalation concentration of sevoflurane) or ordinal scale variables (such as ASA status, New York Heart Association (NYHA) status). Two-sample $t$ test was used for data with normal distributions such as intraoperative and postoperative opioid consumption, and first postoperative flatus time. The two-sample test for binomial proportions or Fisher's exact test were used to analyze the difference between the two groups in the number of vertebral levels surgically treated and postoperative nausea/ vomiting. All tests were one-sided.

\section{Results}

There was no significant difference between groups in major demographic variables, such as age, gender, BMI, history of opioid usage, ASA status, the number of lumbar spine levels treated $(P=0.296)$, surgical time $(P=0.136)$ and general anesthesia duration $(P=0.136)$ (Table 1$)$.

\section{Primary Outcome: VAS Pain Scores for the First 48 h After Surgery}

The result of Mauchly's Test of Sphericity showed that the correlations between each two neighboring time points $(P=0.000)$ were strong. BD result indicated that there were significant differences in VAS scores over time between the two matched groups $(P=0.010)$. From the time line chart (Figure 3), patients in Group E reported 
Table I Patient Demographics

\begin{tabular}{|c|c|c|c|}
\hline Variable & Group G (n=32) Mean \pm SD/N (\%) & Group E $(n=30)$ Mean \pm SD/N (\%) & $P$-value \\
\hline Age (years) & $56.094 \pm 11.372$ & $56.733 \pm 8.690$ & 0.597 \\
\hline Gender (M/F) & $15(46.9 \%) / 17(53.1 \%)$ & $12(40.0 \%) / 18(60.0 \%)$ & 0.585 \\
\hline Height $(\mathrm{cm})$ & $164.938 \pm 6.535$ & $|64.567 \pm 6.97|$ & 0.415 \\
\hline Weight (kg) & $66.14 I \pm 11.677$ & $67.167 \pm 10.648$ & 0.640 \\
\hline BMI & $24.3 I I \pm 4.86 I$ & $23.882 \pm 5.270$ & 0.648 \\
\hline NYHA Status (Norm/I/II) & $20(62.5 \%) / 10(31.3 \%) / 2(6.2 \%)$ & 21 (70.0\%)/8 (26.7\%)/I (3.3\%) & 0.256 \\
\hline ASA Status $(I / I / I I I)$ & $9(28.1 \%) / 13(40.6 \%) / 10$ (31.3\%) & $6(20.0 \%) / 19(63.3 \%) / 5$ (16.7\%) & 0.361 \\
\hline Drinking History & $9(28.1 \%)$ & $7(21.9 \%)$ & 0.667 \\
\hline Smoking History & $8(25.0 \%)$ & $10(33.3 \%)$ & 0.470 \\
\hline History of Opioid Use & 0 & 0 & - \\
\hline General Anesthesia Duration (min) & $245.156 \pm 60.074$ & $253.667 \pm 51.090$ & 0.552 \\
\hline Surgical Duration (min) & $215.975 \pm 59.629$ & $211.500 \pm 51.145$ & 0.755 \\
\hline \multicolumn{4}{|l|}{ Number of spine levels involved segment (ESP block level) } \\
\hline $2 / 3$ Surgical Levels & $15(46.9 \%) / 17(53.1 \%)$ & $19(63.3 \%) / 11$ (36.7\%) & 0.296 \\
\hline $\mathrm{LI} / 2 / 3(\mathrm{~L} 2)$ & I (3.1\%) & $2(6.7 \%)$ & \\
\hline $\mathrm{L} 2 / 3$ (L2) & I (3.1\%) & $0(0 \%)$ & \\
\hline L2/3/4 (L3) & $5(15.6 \%)$ & I (3.3\%) & \\
\hline $\mathrm{L} 3 / 4(\mathrm{~L} 3)$ & $3(9.4 \%)$ & I (3.3\%) & \\
\hline L3/4/5 (L4) & 7 (21.9\%) & $2(6.7 \%)$ & \\
\hline L4/5 (L4) & $3(9.4 \%)$ & $14(46.7 \%)$ & \\
\hline L4/5/SI (L5) & $4(12.5 \%)$ & $6(20.0 \%)$ & \\
\hline L5/SI (L5) & $8(25.0 \%)$ & $4(13.3 \%)$ & \\
\hline
\end{tabular}

Abbreviations: Group G, control group; Group E, treatment group; BMI, body mass index; NYHA, New York Heart Association; ASA, American Society of Anesthesiologists; L, lumbar spine; S, sacral spine.

less pain than patients in Group $\mathrm{G}$ in the early postoperative period, postoperative VAS at $1 \mathrm{~h}, 3 \mathrm{~h}, 6 \mathrm{~h}$ in Group $\mathrm{G}$ and Group $\mathrm{E}$ were $4.250 \pm 1.414$ vs $2.033 \pm 0.718$, $P=0.000, \quad 3.906 \pm 1.14$ vs $2.400 \pm 0.814, \quad P=0.000$, and $3.656 \pm 1.066$ vs $2.767 \pm 0.679, \quad P=0.000$ respectively. Group $\mathrm{G}$ and Group E VAS scores peaked at $1 \mathrm{~h}$ and 12 $h$, respectively, and the peak pain score in Group $G$ is

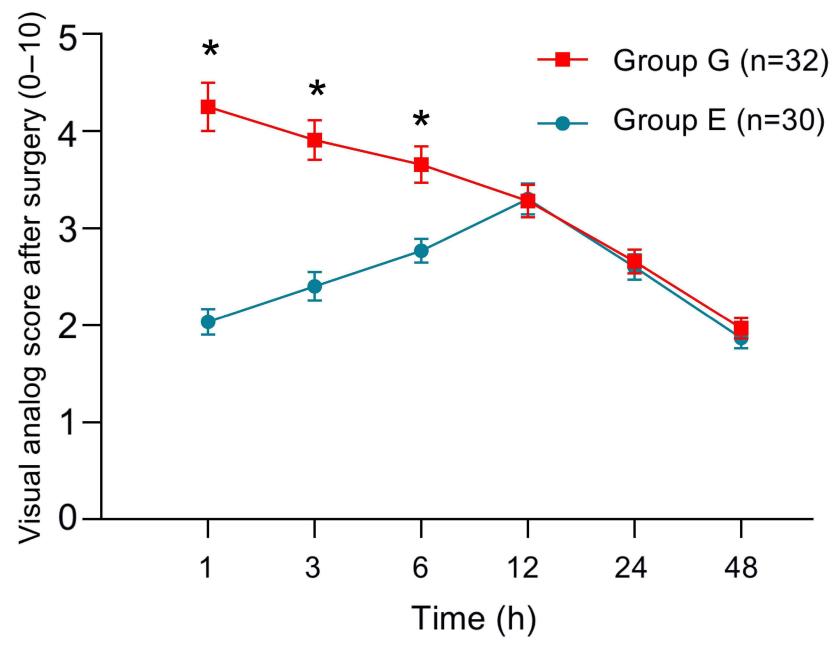

Figure 3 VAS pain score timeline chart. ${ }^{\star} * P<0.05$. Data are presented as mean $\pm S D$. significantly higher than that of Group E $(P=0.002)$. In addition, the OBAS pain satisfaction score of Group E was significantly lower than that of Group $\mathrm{G}(P=0.008)$; lower OBAS score corresponds to a greater benefit from a specific pain management therapy (Table 2).

\section{Secondary Outcomes: Hemodynamics, Analgesics Consumption and Recovery}

Intraoperative hemodynamics were monitored continuously through a radial arterial line. The diastolic blood pressure change $(\Delta \mathrm{DBP})$ and heart rate change $(\Delta \mathrm{HR})$ in Group $\mathrm{G}$ were significantly higher than that of Group $\mathrm{E}\left(P_{\triangle \mathrm{DBP}}=0.000, P_{\Delta \mathrm{HR}}=0.003\right)$ (Table 2$)$.

The anesthetic consumption of Group $G$ was significantly higher than that of Group E (Table 2), including intraoperative inhaled concentration of sevoflurane $(P=0.000)$ and intraoperative sevoflurane consumption $(P=0.000)$. The function of geeglm fitting generalized estimating equations was used to analyze the difference in the number of PCA attempts and opioid consumption (oral morphine milligram equivalent, OME) with time between the two groups, which showed the number of PCA attempts and opioid consumption (OME) were statistically 
Table 2 Intraoperative Hemodynamics and Perioperative Anesthetic/Analgesic Consumption

\begin{tabular}{|c|c|c|c|}
\hline \multirow[t]{2}{*}{ Variable } & \multirow{2}{*}{$\begin{array}{l}\text { Group G }(n=32) \\
\text { Mean } \pm \text { SD }\end{array}$} & \multirow{2}{*}{$\begin{array}{l}\text { Group E }(n=30) \\
\text { Mean } \pm \text { SD }\end{array}$} & \multirow[t]{2}{*}{$P$-value } \\
\hline & & & \\
\hline \multicolumn{4}{|l|}{ Hemodynamics } \\
\hline$\triangle \mathrm{SBP}(\mathrm{mmHg})$ & $8.000 \pm 4.500$ & $7.500 \pm 4.000$ & 0.177 \\
\hline$\triangle \mathrm{DBP}(\mathrm{mmHg})$ & $6.000 \pm 4.000$ & $3.500 \pm 3.000$ & 0.000 \\
\hline$\Delta \mathrm{HR}(\mathrm{bpm})$ & $3.000 \pm 3.000$ & $1.500 \pm 2.750$ & 0.003 \\
\hline OBAS at $24 \mathrm{~h}$ & $3.000 \pm 2.000$ & $2.000 \pm 1.000$ & 0.008 \\
\hline \multicolumn{4}{|l|}{ Anesthetic and Analgesic Consumption } \\
\hline Intraoperative Inhalational Sevoflurane Concentration & $2.500 \pm 0.625$ & $1.500 \pm 0.500$ & 0.000 \\
\hline Intraoperative Sevoflurane Consumption (mL) & $79.200 \pm 23.625$ & $46.800 \pm 17.100$ & 0.000 \\
\hline Intraoperative Opioid Consumption (OME) & $41.300 \pm 5.820$ & $22.200 \pm 4.710$ & 0.000 \\
\hline Postoperative Opioid Consumption (OME) at $0-12 \mathrm{~h}$ & $17.800 \pm 2.100$ & $10.300 \pm 3.700$ & 0.000 \\
\hline Postoperative Opioid Consumption (OME) at I2-24 h & $16.900 \pm 1.470$ & $16.700 \pm 2.770$ & 0.692 \\
\hline Postoperative Opioid Consumption (OME) at 24-48 h & $18.000 \pm 3.960$ & $18.400 \pm 4.400$ & 0.721 \\
\hline Total Postoperative Opioid Consumption (OME) at $0-48 \mathrm{~h}$ & $52.763 \pm 6.544$ & $45.547 \pm 9.395$ & 0.001 \\
\hline \multicolumn{4}{|l|}{ The number of PCA Attempts } \\
\hline The number of PCA Attempts at $0-12 \mathrm{~h}$ & $1.660 \pm 1.070$ & $0.633 \pm 0.809$ & 0.000 \\
\hline The number of PCA Attempts at $12-24 \mathrm{~h}$ & $1.030 \pm 0.595$ & $0.933 \pm 0.828$ & 0.463 \\
\hline The number of PCA Attempts at $24-48 \mathrm{~h}$ & $0.312 \pm 0.535$ & $0.567 \pm 0.626$ & 0.081 \\
\hline The total number of PCA Attempts at $0-48 \mathrm{~h}$ & $2.500 \pm 2.000$ & $2.000 \pm 3.750$ & 0.037 \\
\hline Number of Rescue Analgesia Received & $1.000 \pm 2.000$ & $1.000 \pm 1.000$ & 0.215 \\
\hline Number of Patients Using Pethidine & 0 & 0 & - \\
\hline
\end{tabular}

Abbreviations: Group G, control group; Group E, treatment group; SBP, systolic blood pressure; DBP, diastolic blood pressure; HR, heart rate; OBAS, overall benefit of analgesia score; OME, oral morphine milligram equivalent; PCA, patient-controlled analgesia.

different over time between the two groups as interactions exist between each anesthesia technique (without vs with ESP block) and the timing for PCA attempts $(P=0.000)$ and opioid consumption (OME, $P=0.000$ ). The opioid consumption (OME) in Group $\mathrm{G}$ was significantly higher than that in Group E both intraoperatively $(P=0.000)$ and postoperatively $(P=0.0005)$. Specifically, the opioid consumption (OME) and the numbers of PCA attempts in Group $G$ was significantly higher than that in Group E ( $P=0.000$ and $P=0.000$ respectively) at $0-12 \mathrm{~h}$ (Table 2$)$.
In addition, the time to first postoperative flatus in Group E was significantly earlier than that in Group $\mathrm{G}(P=0.014)$ (Table 3).

\section{Discussion}

Lumbar spine surgery is frequently performed to relieve pain and provide functional improvement in patients with spinal stenosis and degenerative disc disease. ${ }^{18,19}$ During the surgery, the paraspinal muscles are dissected away from the spinous process and lamina to the lateral side of

Table 3 Postoperative Recovery

\begin{tabular}{|c|c|c|c|}
\hline \multirow[t]{2}{*}{ Variable } & \multirow{2}{*}{$\begin{array}{l}\text { Group G }(n=32) \\
\text { Mean } \pm \text { SD/N (\%) }\end{array}$} & \multirow{2}{*}{$\begin{array}{l}\text { Group E }(n=30) \\
\text { Mean } \pm S D / N(\%)\end{array}$} & \multirow[t]{2}{*}{$P$-value } \\
\hline & & & \\
\hline Time to First Postoperative Flatus & $39.875 \pm 14.494$ & $32.000 \pm 15.682$ & 0.014 \\
\hline Hospital Stay (h) & $395.344 \pm 77.994$ & $398.767 \pm 92.562$ & 0.562 \\
\hline Hospitalization Expenses (yuan, RMB) & $63,246.650 \pm 23,769.970$ & $57,124.190 \pm \mid 4,455.210$ & 0.111 \\
\hline Postoperative Nausea & II (34.4\%) & $6(5.0 \%)$ & 0.205 \\
\hline Postoperative Vomiting & $5(15.6 \%)$ & $4(13.3 \%)$ & 1.000 \\
\hline Postoperative Dizzines & I (3.1\%) & $0(0 \%)$ & - \\
\hline Postoperative Irritability & $0(0 \%)$ & $0(0 \%)$ & - \\
\hline
\end{tabular}

Abbreviations: Group G, control group; Group E, treatment group. 
the facet joint. Mechanical and thermal trauma during surgery may cause muscle ischemia and damage to nerves innervating the paraspinal muscles. ${ }^{9,20}$ Severe postoperative pain is common. ${ }^{9,18-20}$ Opioid agonists are the mainstay of treatment for perioperative pain, but their administration slows down normal gastrointestinal motility, increases the incidence of respiratory complications, and prolongs hospital stay. ${ }^{21}$ Enhanced recovery after surgery protocols frequently include the use of regional anesthesia techniques to minimize opioid analgesics whenever possible. $^{22,23}$ Our study showed that the ESP block provided improved analgesia with significantly lower VAS scores at rest at 1,3 , and $6 \mathrm{~h}$ postoperatively, decreased intraoperative and postoperative opioid consumption and decreased intraoperative sevoflurane requirements. OBAS as an indicator of patient satisfaction for pain control, ${ }^{14}$ was also shown to be better in Group E compared to group G. Finally, effective analgesia via opioid-reducing ESP block placed preoperatively was shown to accelerate bowel function recovery postoperatively.

Our study adds to a small field of randomized control trials studying the efficacy of ESP block for analgesia in lumbar spine surgery. Bilateral ESP block for open lumbar decompression surgery was assessed through a singlecenter randomized control study of 60 patients. ${ }^{11}$ Lower pain scores at rest and with activity, as well as decreased opioid consumption were observed for $24 \mathrm{~h}$ after surgery in patients with ESP block. ${ }^{11}$ Another single-center randomized control study of 40 patients on the effectiveness of bilateral ESP block in lumbar spine surgery had results similar to our study, with lower pain scores for the first 6 $\mathrm{h}$ after surgery, less rescue analgesics and lower opioid consumption at $24 \mathrm{~h}$ in the patients with ESP block. ${ }^{10}$ A multicenter trial of 80 patients investigating the efficacy of bilateral lumbar ESP block for posterior lumbar interbody fusion surgery is currently recruiting; opioid consumption at $24 \mathrm{~h}$ is the primary outcome. This multicenter trial may add to our understanding of the contribution of ESP block for analgesia in lumbar spine surgery. ${ }^{9}$

The ESP block performed in the lumbar spine region by depositing local anesthetics in the fascial plane superficial to the transverse process directly and consistently targets the dorsal rami of the spinal nerves, thus providing sensory input to the posterior wall of the trunk. ${ }^{1,2,4,6}$ The spread of local anesthetics into the paravertebral or epidural space, albeit controversial and not consistently observed, may provide visceral pain control in addition to somatic analgesia, but adds the potential risk of lower extremity weakness. ${ }^{24-26}$ However, ESP block has been used for lower extremity hip, femur and knee procedures and there has been no more motor weakness or interference with physical therapy when compared to other regional anesthesia techniques, such as lumbar plexus and quadratus lumborum block. ${ }^{27,28}$ In summary, we would not anticipate ESP block to cause motor weakness or interfere with early ambulation.

Concerns remain that the analgesic effect of preincision ESP block for spine surgery may be diminished because of the proximity of the injection site to the surgical incision and the potential for local anesthetics to be washed away. The same pre-incision technique has been used in other studies that have confirmed the analgesic effects of ESP block for spine surgery, ${ }^{10,11}$ with a similar time interval between block placement and surgical incision. ${ }^{11}$ In addition, ultrasound-guided modified thoracolumbar interfascial plane (TLIP) block, a similar fascial plane block to the ESP block, has been shown to provide effective analgesia even when performed immediately before surgical incision under general anesthesia. ${ }^{29,30}$ In this study, the surgical incision extended from midline to the articular process but did not extend to the transverse process. Also, incision did not occur immediately after the ESP block was performed; at least half-an-hour elapsed, providing enough time for local anesthetics to spread to the targeted location and bind to receptors. In the future, it would be of interest to design a study comparing the efficacy of ESP blocks performed at different time intervals before surgical incision.

Alternative regional techniques for analgesia for lumbar spine surgery have been described and include epidural anesthesia, paravertebral nerve block (PVB), retrolaminar block (RLB), TLIP block, and local anesthetic wound infiltration. Compared to neuraxial blocks, the ESP block avoids serious neuraxial complications such as dural puncture, epidural hematoma, and hypotension. ${ }^{31,32}$ PVB provides more reliable ventral ramus blockade, helpful when anterior surgical exposure is utilized, and sympathetic trunk blockade and thus greater visceral pain control than ESP block, but is associated with more risks for serious complications such as pneumothorax in the thoracic spine levels, hypotension, nerve injury, and it can be technically challenging to perform. ${ }^{33}$ The injectate of RLB could diffuse into the paravertebral space, epidural space, and intervertebral foramina. ${ }^{33}$ Although the two types of TLIP block method can provide effective analgesia for patients 
undergoing lumbar discectomy, ${ }^{29,30}$ this block can be technically challenging because in some patients identification of the paraspinal muscles may prove difficult as these separate muscles often appear as a single larger one in some patients. ${ }^{34}$ Local anesthetic wound infiltration is common but the effects are often short-lived. ${ }^{35}$ Potential benefits of the lumbar ESP block include the ease of performance with clear landmarks for ultrasound anatomy. In addition, the technique is very safe, since the target site for injection is a muscular plane and there is practically no risk for mechanical nerve contact. ${ }^{24,28}$ Risk of injury to neuraxis is also very low.

In this study, the analgesic effects of a bilateral single injection ESP block with long-acting local anesthetics diminished after $12 \mathrm{~h}$. Multiple options are available for providing a longer duration of pain control. Patients may benefit from repeated injections. A continuous nerve block catheter for inpatients, or an indwelling catheter connected to a disposable pump for outpatients may significantly prolong perioperative pain control, accelerate recovery and shorten length of hospital stay. However, concerns about the potential risk for wound infections because of the close proximity of the surgical incision to the ESP block site will limit the application of ESP catheters for lumbar spine surgeries. Liposomal bupivacaine is another option for prolonging one-time injections; however, its potential duration of $72 \mathrm{~h}$ has not been universally established and its cost is prohibitive in many settings. Several local anesthetic adjuvants such as dexamethasone, ${ }^{36}$ dexmedetomidine, ${ }^{37}$ compound betamethasone ${ }^{38}$ and methylprednisolone acetate ${ }^{39,40}$ have been used to prolong the analgesic effects of single-shot blocks from hours to days. Such adjuvants have the potential to prolong ESP blocks; further investigations of this block for lumbar spine surgery that include local anesthetic adjuvants would be helpful.

There are several limitations of this study. The blinding is not complete as neither the anesthesiologist who prepared all the medication, performed all the general anesthesia management and ESP blocks, nor the surgeons were blinded to the randomization. However, all patients were unaware of the grouping as blocks were performed after general anesthesia. In addition, all perioperative management and postoperative assessment were performed by two certified nurse investigators who were unaware of group allocation. With respect to the block itself, we did not measure the exact spread of local anesthetic after injection and were not able to assess the dermatomal coverage post block placement. Unfortunately, because our hospital does not use acetaminophen for analgesia, it was not included in the multimodal analgesic protocol for this study; parecoxib, a selective COX-2 inhibitor, was used instead. Intravenous acetaminophen is not commonly used perioperatively in China, and oral acetaminophen is typically only used as an antipyretic and infrequently as a perioperative analgesic. Ideally, both would have been used to maximize their opioid-sparing and synergistic analgesic effects with opioids. Also, because our surgeons prefer that their patients are not mobilized for the first 48 $h$ postoperatively due to concerns for wound dehiscence and inadequate pain control, we were not able to monitor the effects of ESP on VAS dynamic pain scores or the number of PCA attempts and opioid consumption with activity. Collecting this information would be helpful in future studies. Timing of the subsequent initiation of mobilization was not documented, but there were no reports of quadriceps weakness or difficulty with physical therapy in any study patients. In addition, the patients in this study underwent only lumbar laminoplasty of two to three levels, so the benefits of this block for other lumbar surgeries is not confirmed. Moreover, this study focused on short-term outcomes for the first $48 \mathrm{~h}$ after surgery, and long-term outcomes such as the efficacy of the ESP block to prevent persistent postsurgical pain remains unknown. A larger-scale study, ideally a multicenter, prospective study, including patients for different types of spine procedures, would be better for systemically assessing the effectiveness of the ESP block for spine surgery.

\section{Conclusions}

The ultrasound-guided bilateral ESP block can provide effective analgesia, decrease perioperative opioid consumption, improve patient satisfaction with perioperative pain management and is beneficial for the recovery of gastrointestinal function in patients undergoing lumbar laminoplasty.

\section{Data Sharing Statement}

No further data will be shared besides what is included in this manuscript.

\section{Ethics Approval and Consent to Participate}

The study was conducted with Institutional Review Board approval from the Second Hospital of Shandong University in China (number KYLL-2018(LW)031), and the guidelines outlined in the Declaration of Helsinki were 
followed. Written informed consent was obtained from all study participants.

\section{Consent for Publication}

Obtained.

\section{Acknowledgments}

The authors would like to thank Dr Yongtao Sun for study design, Miss Shuai $\mathrm{Hu}$ and Miss Yingchao Zhang for postoperative patient follow-up, and Miss Qi Han for data collection. Haizhu Tan and Jinlei Li are cocorresponding authors.

\section{Author Contributions}

All authors made a significant contribution to the work reported, whether that is in the conception, study design, execution, acquisition of data, analysis and interpretation, or in all these areas; took part in drafting, revising or critically reviewing the article; gave final approval of the version to be published; have agreed on the journal to which the article has been submitted; and agree to be accountable for all aspects of the work.

\section{Funding}

This present study was supported by the National Natural Science Foundation of China (11801540), the Natural Science Foundation of Guangdong (2017A030310572), and Shantou Science and Technology Project (2018) to Professor Haizhu Tan.

\section{Disclosure}

The authors report no conflicts of interest in this work.

\section{References}

1. Yörükoğlu HU, Içli D, Aksu C, Cesur S, Kuş A, Gürkan Y. Erector spinae block for postoperative pain management in lumbar disc hernia repair. $J$ Anesth. 2021;35(3):420-425. doi:10.1007/s00540-021-02920-0

2. Goel VK, Chandramohan M, Murugan C, et al. Clinical efficacy of ultrasound guided bilateral erector spinae block for single level lumbar fusion surgery: a prospective, randomized, case-control study. Spine J. 2021. doi:10.1016/j.spinee.2021.06.015

3. Saadawi M, Layera S, Aliste J, Bravo D, Leurcharusmee P, De Tran Q. Erector spinae plane block: a narrative review with systematic analysis of the evidence pertaining to clinical indications and alternative truncal blocks. J Clin Anesth. 2021;68:110063. doi:10.1016/j. jclinane.2020.110063

4. Chin KJ, El-Boghdadly K. Mechanisms of action of the erector spinae plane (ESP) block: a narrative review. Can J Anaesth. 2021;68 (3):387-408. doi:10.1007/s12630-020-01875-2
5. Qiu Y, Zhang T-J, Hua Z. Erector spinae plane block for lumbar spinal surgery: a systematic review. J Pain Res. 2020;13:1611-1619. doi:10.2147/JPR.S256205

6. Harbell MW, Seamans DP, Koyyalamudi V, Kraus MB, Craner RC, Langley NR. Evaluating the extent of lumbar erector spinae plane block: an anatomical study. Reg Anesth Pain Med. 2020;45 (8):640-644. doi:10.1136/rapm-2020-101523

7. Cesur S, Yayik AM, Ozturk F, Ahiskalioglu A. Ultrasound-guided low thoracic erector spinae plane block for effective postoperative analgesia after lumbar surgery: report of five cases. Cureus. 2018;10: e3603. doi:10.7759/cureus.3603

8. Ueshima H, Inagaki M, Toyone T, Otake H. Efficacy of the erector spinae plane block for lumbar spinal surgery: a retrospective study. Asian Spine J. 2019;13:254-257. doi:10.31616/asj.2018.0114

9. Breebaart MB, Van Aken D, De Fré O, et al. A prospective randomized double-blind trial of the efficacy of a bilateral lumbar erector spinae block on the $24 \mathrm{~h}$ morphine consumption after posterior lumbar inter-body fusion surgery. Trials. 2019;20:441. doi:10.1186/s13063-019-3541-y

10. Singh S, Chaudhary NK. Bilateral Ultasound guided erector spinae plane block for postoperative pain management in lumbar spine surgery: a case series. J Neurosurg Anesthesiol. 2019;31(3):354. doi:10.1097/ANA.0000000000000518

11. Yayik AM, Cesur S, Ozturk F, et al. Postoperative analgesic efficacy of the ultrasound-guided erector spinae plane block in patients undergoing lumbar spinal decompression surgery: a randomized controlled study. World Neurosurg. 2019;126:e779-e785. doi:10.1016/j. wneu.2019.02.149

12. Kowark A, Rossaint R, Puhringer F, et al. Emergence times and airway reactions during general anaesthesia with remifentanil and a laryngeal mask airway: a multicentre randomised controlled trial. Eur J Anaesthesiol. 2018;35:588-597. doi:10.1097/EJA.0000000000000852

13. Zhao X, Jin YW, Li HB, Wang ZG, Feng H, Feng C. Effects of maintaining intravenous infusion of remifentanil or propofol on anesthesia and palinesthesia during anesthesia and analepsia. Genet Mol Res. 2014;13:2865-2872. doi:10.4238/2014.February.13.8

14. Lehmann N, Joshi GP, Dirkmann D, et al. Development and longitudinal validation of the overall benefit of analgesia score: a simple multi-dimensional quality assessment instrument. $\mathrm{Br} J$ Anaesth. 2010;105(4):511-518. doi:10.1093/bja/aeq186

15. Wang Z, Goonewardene LA. The use of MIXED models in the analysis of animal experiments with repeated measures data. Can J Anim Sci. 2004;84:1-11. doi:10.1002/1096-9888(200006)35:63.0.CO;2-I

16. Grieve AP. Tests of sphericity of normal-distributions and the analysis of repeated measures designs. Psychometrika. 1984;49:257-267. doi:10.1007/BF02294176

17. Pan W, Tian Y, Wang X, Zhang H. Ball divergence: nonparametric two sample test. Ann Stat. 2018;46:1109-1137. doi:10.1214/17-AOS1579

18. Zhi-Jun H, Fang X-Q, Zhou Z-J, Wang J-Y, Zhao F-D, Fan S-W. Effect and possible mechanism of muscle-splitting approach on multifidus muscle injury and atrophy after posterior lumbar spine surgery. J Bone Joint Surg Am. 2013;95(24):e192(1-9). doi:10.2106/JBJS. L.01607

19. Archer KR, Devin CJ, Vanston SW, et al. Cognitive-behavioral-based physical therapy for patients with chronic pain undergoing lumbar spine surgery: a randomized controlled trial. J Pain. 2016;17 (1):76-89. doi:10.1016/j.jpain.2015.09.013

20. Fu C-J, Chen W-C, Lu M-L, Cheng C-H, Niu -C-C. Comparison of paraspinal muscle degeneration and decompression effect between conventional open and minimal invasive approaches for posterior lumbar spine surgery. Sci Rep. 2020;10(1):14635. doi:10.1038/ s41598-020-71515-8

21. Muller-Lissner S, Bassotti G, Coffin B, et al. Opioid-induced constipation and bowel dysfunction: a clinical guideline. Pain Med. 2017;18:1837-1863. doi:10.1093/pm/pnw255 
22. Gustafsson UO, Scott MJ, Schwenk W, et al. Guidelines for perioperative care in elective colonic surgery: enhanced Recovery After Surgery (ERAS $(\circledR))$ Society recommendations. World J Surg. 2013;37:259-284. doi:10.1007/s00268-012-1772-0

23. Tan M, Law LS, Gan TJ. Optimizing pain management to facilitate enhanced recovery after surgery pathways. Can J Anaesth. 2015;62:203-218. doi:10.1007/s12630-014-0275-x

24. Kot P, Rodriguez P, Granell M, et al. The erector spinae plane block: a narrative review. Korean $J$ Anesthesiol. 2019;72:209-220. doi:10.4097/kja.d.19.00012

25. Tulgar S, Selvi O, Senturk O, Ermis MN, Cubuk R, Ozer Z. Clinical experiences of ultrasound-guided lumbar erector spinae plane block for hip joint and proximal femur surgeries. $J$ Clin Anesth. 2018;47:5-6. doi:10.1016/j.jclinane.2018.02.014

26. Karaca O, Pinar HU. Is high dose lumbar erector spinae plane block safe? J Clin Anesth. 2020;62:109721. doi:10.1016/j.jclinane.20 20.109721

27. Tulgar S, Kose HC, Selvi O, et al. Comparison of ultrasound-guided lumbar erector spinae plane block and transmuscular quadratus lumborum block for postoperative analgesia in hip and proximal femur surgery: a prospective randomized feasibility study. Anesthesia Essays Res. 2018;12:825-831. doi:10.4103/aer.AER_142_18

28. Tulgar S, Selvi O, Senturk O, Ermis MN, Cubuk R, Ozer Z. Clinical experiences of ultrasound-guided lumbar erector spinae plane block for hip joint and proximal femur surgeries. $J$ Clin Anesth. 2018;47:5-6. doi:10.1016/j.jclinane.2018.02.014

29. Li C, Jia J, Qin Z, Tang Z. The use of ultrasound-guided modified thoracolumbar interfascial plane (TLIP) block for multi-level lumbar spinal surgery. J Clin Anesth. 2018;46:49-51. doi:10.1016/j. jclinane.2018.01.018

30. Ohgoshi Y, Namiki R, Kori S, Matsukawa M. The use of ultrasound-guided thoracolumbar interfascial plane block for multi-level lumbar spinal surgery. J Clin Anesth. 2017;37:162. doi:10.1016/j.jclinane.2016.12.025

31. Adhikary SD, Pruett A, Forero M, Thiruvenkatarajan V. Erector spinae plane block as an alternative to epidural analgesia for post-operative analgesia following video-assisted thoracoscopic surgery: a case study and a literature review on the spread of local anaesthetic in the erector spinae plane. Indian $J$ Anaesth. 2018;62:75-78. doi:10.4103/ija.IJA_693_17
32. Nagaraja PS, Ragavendran S, Singh NG, et al. Comparison of continuous thoracic epidural analgesia with bilateral erector spinae plane block for perioperative pain management in cardiac surgery. Ann Card Anaesth. 2018;21:323-327. doi:10.4103/aca.ACA_16_18

33. Onishi E, Toda N, Kameyama Y, Yamauchi M. Comparison of clinical efficacy and anatomical investigation between retrolaminar block and erector spinae plane block. Biomed Res Int. 2019;2019:2578396. doi:10.1155/2019/2578396

34. Ammar MA, Taeimah M. Evaluation of thoracolumbar interfascial plane block for postoperative analgesia after herniated lumbar disc surgery: a randomized clinical trial. Saudi J Anaesth. 2018;12:559-564. doi:10.4103/sja.SJA_177_18

35. Ince I, Atalay C, Ozmen $\mathrm{O}$, et al. Comparison of ultrasound-guided thoracolumbar interfascial plane block versus wound infiltration for postoperative analgesia after single-level discectomy. J Clin Anesth. 2019;56:113-114. doi:10.1016/j.jclinane.2019.01.017

36. Zorrilla-Vaca A, Li J. Dexamethasone injected perineurally is more effective than administered intravenously for peripheral nerve blocks: a meta-analysis of randomized controlled trials. Clin J Pain. 2018;34:276-284. doi:10.1097/AJP.0000000000000519

37. Hu X, Li J, Zhou R, et al. Dexmedetomidine added to local anesthetic mixture of lidocaine and ropivacaine enhances onset and prolongs duration of a popliteal approach to sciatic nerve blockade. Clin Ther. 2017;39:89-97.e81. doi:10.1016/j.clinthera.2016.11.011

38. Li J, Li L, Zhang X, et al. Paravertebral block with compound betamethasone in laparoscopic cholecystectomy: a double-blind randomized controlled trial. Cureus. 2019;11:e6023. doi:10.7759/ cureus. 6023

39. Li J, Dai F, Ona Ayala KE, Zhou B, Schonberger RB, Sharma A. Transmuscular quadratus lumborum and lateral femoral cutaneous nerve block in total hip arthroplasty. Clin J Pain. 2021;37 (5):366-371. doi:10.1097/AJP.0000000000000923

40. Li J, Perese F, Rubin LE, Carlyle D. Effective pain management after total hip arthroplasty in a sickle cell patient emphasizing dexamethasone sodium phosphate/methylprednisolone acetate administered via a peripheral nerve blockade: a case report. $A$ A Pract. 2019;12:171-175. doi:10.1213/XAA.0000000000000877
Journal of Pain Research

\section{Publish your work in this journal}

The Journal of Pain Research is an international, peer reviewed, open access, online journal that welcomes laboratory and clinical findings in the fields of pain research and the prevention and management of pain. Original research, reviews, symposium reports, hypothesis formation and commentaries are all considered for publication. The manuscript management system is completely online and includes a very quick and fair peer-review system, which is all easy to use. Visit http:// www.dovepress.com/testimonials.php to read real quotes from published authors. 